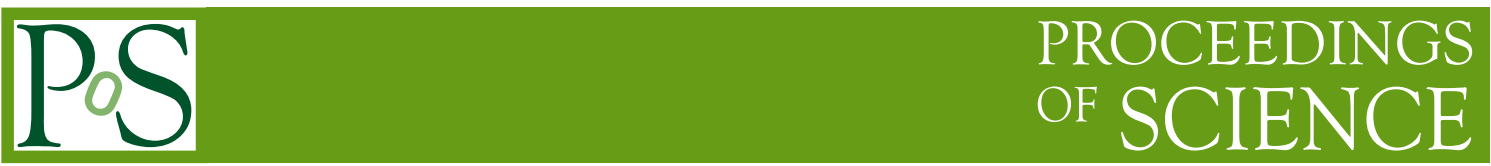

\title{
Lepton Universality tests using semileptonic b-hadron decays
}

\section{Beatriz García Plana ${ }^{a, *}$ on behalf of the LHCb Collaboration}

${ }^{a}$ Insituto Galego de Física de Altas Enerxías, Universidade de Santiago de Compostela, Santiago, Spain

E-mail: beatriz.garcia.plana@cern.ch

Lepton Flavour Universality tests are powerful probes of the Standard Model and, in turn, of New Physics effects. Semileptonic b-hadron decays provide an excellent laboratory for testing Lepton Flavour Universality. A review of these tests performed using data collected by the LHCb experiment in 2011 and 2012 at centre-of-mass energies of 7 and $8 \mathrm{TeV}$ is presented.

40th International Conference on High Energy physics - ICHEP2020

July 28 - August 6, 2020

Prague, Czech Republic (virtual meeting)

\footnotetext{
${ }^{*}$ Speaker
} 


\section{Introduction}

In the Standard Model (SM) of particle physics, the electroweak coupling of the gauge bosons to leptons is independent of the lepton flavour. This property is called Lepton Flavour Universality (LFU), and it implies that the amplitude of the processes involving these particles are identical, neglecting phase space and helicity suppression effects. LFU is enforced in the SM by construction, therefore any violation of it would be a clear sign of physics beyond the SM. Many extensions of the SM include new contributions that involve the third generations of quarks and leptons. Therefore, if there is hierarchy in the strength of the coupling of New Physics (NP) to the leptons of different generations, a comparison between semitauonic and semimuonic b-hadron decays is sensitive to these new contributions.

\section{Semileptonic decays}

Measurements of ratios of branching fractions represent a powerful test of the SM, as the theoretical uncertainties cancel in the predictions, and experimental systematic uncertainties are greatly reduced. In particular, LFU tests are done by means of the observable $R\left(\mathcal{H}_{c}\right)$, given by:

$$
R\left(\mathcal{H}_{c}\right)=\frac{\mathcal{B}\left(\mathcal{H}_{b} \rightarrow \mathcal{H}_{c} \tau v_{\tau}\right)}{\mathcal{B}\left(\mathcal{H}_{b} \rightarrow \mathcal{H}_{c} \mu v_{\mu}\right)}
$$

where $\mathcal{H}_{b}=B^{0}, B_{(c)}^{+}, \Lambda_{b}^{0}, B_{s}^{0}$ and $\mathcal{H}_{c}=D^{*}, D^{0}, D^{+}, D_{s}^{(*)+}, \Lambda_{c}^{(*)-}, J / \psi$. The processes involved here are tree level transitions mediated by a $W$ boson, and they occur with large probability in the SM. At LHCb [1, 2], the kind of measurements needed to probe LFU are extremely challenging, since the centre-of-mass frame in which the reaction $g g \rightarrow b \bar{b}$ is produced is unknown. Besides, many more particles are produced in the event and, as a consequence, there is a large combinatorial background. Further, neutrinos are present in the decay chain of interest, whose momentum is not measured. Therefore, some approximations have to be made to estimate the momentum of the $\mathcal{H}_{b}$ hadron. In the following sections, the specific procedure that is applied in each case is described. The results were obtained by using data collected by the LHCb detector in 2011 and 2012 at a centre-of-mass energies of 7 and $8 \mathrm{TeV}$.

\section{3. $R\left(D^{*+}\right)$ muonic at $\mathbf{L H C b}$}

The first measurement of $R\left(D^{*+}\right)$ in a hadron collider was published in 2015 by $\mathrm{LHCb}$ [5]. The $\tau^{-}$ candidate is reconstructed in its muonic mode: $\tau^{-} \rightarrow \mu^{-} \bar{v}_{\mu} \nu_{\tau}$, hence the signal channel has the same visible final state as the normalisation channel. The observable is given by:

$$
R\left(D^{*+}\right)=\frac{\mathcal{B}\left(\bar{B}^{0} \rightarrow D^{*+} \tau^{-} \bar{v}_{\tau}\right)}{\mathcal{B}\left(\bar{B}^{0} \rightarrow D^{*+} \mu^{-} \bar{v}_{\mu}\right)},
$$

where the $D^{*+}$ candidate is reconstructed through $D^{*+} \rightarrow D^{0}\left(\rightarrow K^{-} \pi^{+}\right) \pi^{+}$. A three-dimensional fit is performed in the most discriminating variables, in order to measure the yields of the signal, normalisation and background components. These variables are the squared missing mass, $m_{\text {miss }}^{2}$, the energy of the muon in the $B$-meson centre-of-mass system, $E_{\mu}^{*}$, and the squared four momentum 
of the dilepton system, $q^{2}$. The fit results are shown in Fig.1. The measurement is $R\left(D^{*+}\right)=$ $0.336 \pm 0.027$ (stat) \pm 0.030 (syst), which differs by $2.1 \sigma$ compared to the SM. The dominant systematic uncertainty in this analysis comes from the size of the simulated sample. The next largest contribution towards the systematic uncertainty is due to the modelling of the background from hadrons misidentified as muons also gives an important systematic uncertainty contribution.
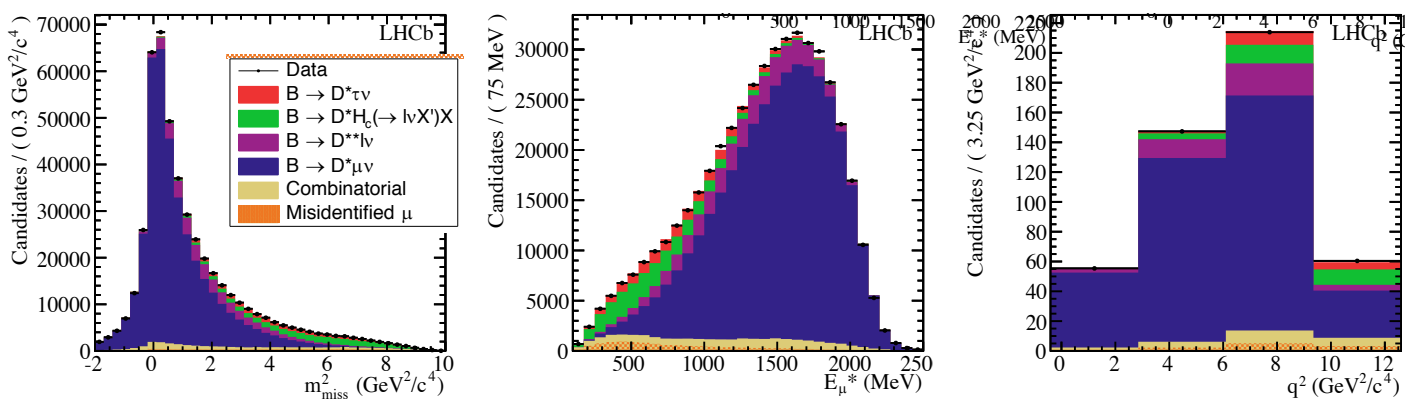

Figure 1: From [5]. Distributions of $m_{m i s s}^{2}$ (left), $E_{\mu}^{*}$ (middle) and $q^{2}$ (right) of data and the projections of the fit model. The signal distributions are shown in red and the normalisation in blue.

\section{4. $R\left(D^{*-}\right)$ hadronic at $\mathbf{L H C b}$}

In 2017, LHCb performed a measurement of $R\left(D^{*-}\right)$ where the $\tau$ is reconstructed in its hadronic mode, consisting of the overlapping signal decay mode $\tau^{-} \rightarrow \pi^{-} \pi^{+} \pi^{-}\left(\pi^{0}\right) v_{\tau}[6,7]$. The $D^{*-}$ meson is reconstructed through the $D^{*-} \rightarrow \bar{D}^{0}\left(\rightarrow K^{+} \pi^{-}\right) \pi^{-}$decay chain. The $B$ decays involved in this analysis are semileptonic decays without leptons in the visible final state, and so the contribution from normal semileptonic decays is zero. In this decay, two neutrinos are missing, hence approximations are needed in order to estimate the $B$ and $\tau$ momenta, which are determined with good precision, up to a two-fold ambiguity each one. The strategy followed in this analysis in order to obtain $R\left(D^{*-}\right)$ is to measure the ratio $\mathcal{K}\left(D^{*-}\right)$, defined as:

$$
\begin{aligned}
\mathcal{K}\left(D^{*-}\right)=\frac{\mathcal{B}\left(B^{0} \rightarrow D^{*-} \tau^{+} v_{\mu}\right)}{\mathcal{B}\left(B^{0} \rightarrow D^{*-} \pi^{+} \pi^{-} \pi^{+}\right)}= & \\
= & \frac{\mathbf{N}_{\text {sig }}}{\mathbf{N}_{\text {norm }}} \times \frac{\epsilon_{\text {norm }}}{\epsilon_{\text {sig }}} \times \frac{1}{\mathcal{B}\left(\tau^{+} \rightarrow \pi^{+} \pi^{-} \pi^{+}\left(\pi^{0}\right) \bar{v}_{\tau}\right)},
\end{aligned}
$$

where $N_{\text {sig }}=N_{B^{0} \rightarrow D^{*-} \tau^{+} v_{\tau}}$ and $N_{\text {norm }}=N_{B^{0} \rightarrow D^{*-3} \pi}$, analogously with the efficiencies. The $\mathcal{K}\left(D^{*-}\right)$ is multiplied subsequently by two external branching fraction inputs that are known with good precision [3]:

$$
R\left(D^{*-}\right)=\mathcal{K}\left(D^{*-}\right) \times \frac{\mathcal{B}\left(B^{0} \rightarrow D^{*-} \pi^{+} \pi^{-} \pi^{+}\right)}{\mathcal{B}\left(B^{0} \rightarrow D^{*-} \mu^{+} v_{\mu}\right)} .
$$

The normalisation channel is chosen such that it shares the same visible final state as the signal. As a result, most of the experimental uncertainties (particle identification or trigger efficiencies) cancel out in the ratio.

One of the major challenges of the analysis was to reduce the largest background contributions from $B \rightarrow D^{*-} 3 \pi X$ decays, where the three pions are produced at the $B$ vertex. To achieve this, the 

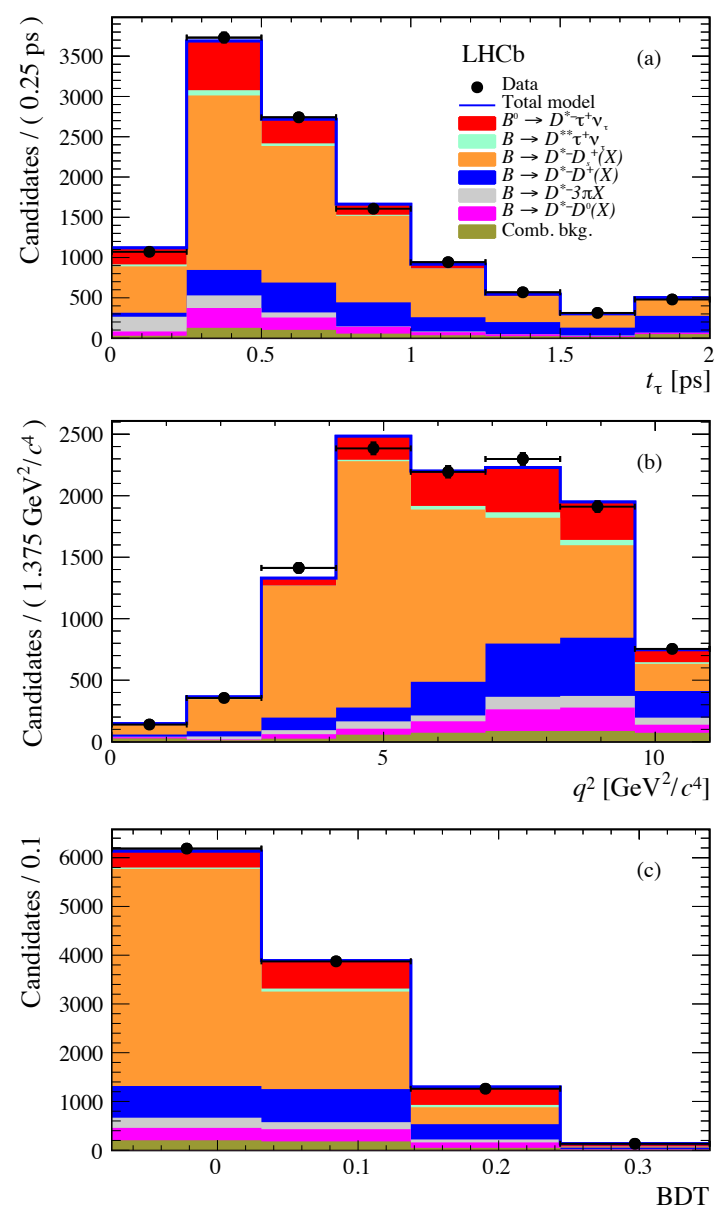

(a)

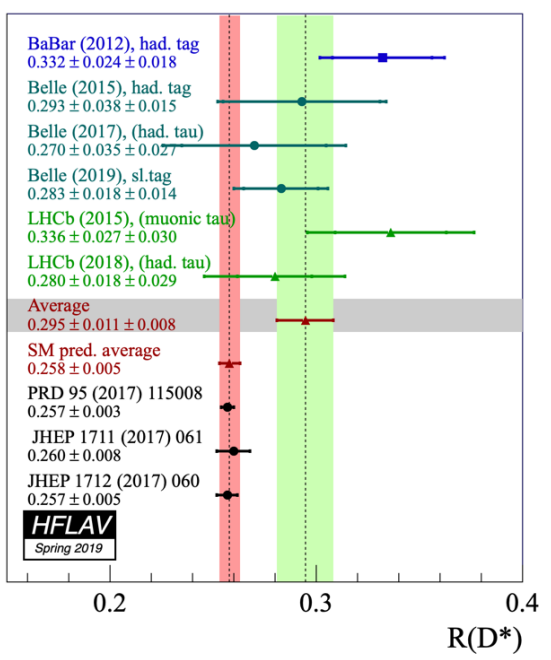

(b)

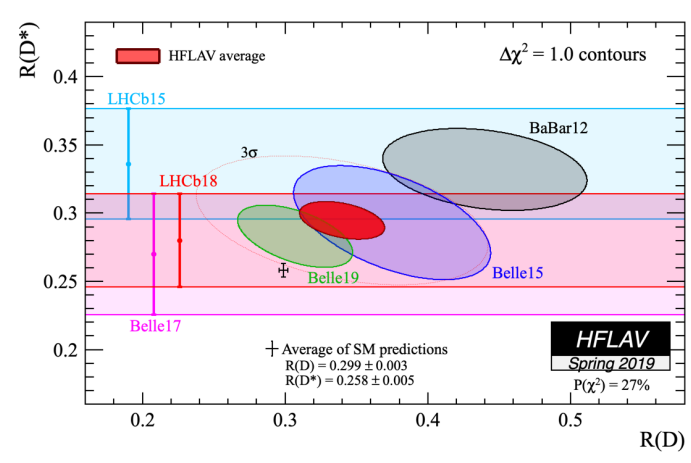

(c)

Figure 2: (a) From [6]. Distribution of $t_{\tau}$ (top), $q^{2}$ (middle) and BDT (bottom). (b) From [4]. Comparison of the $R\left(D^{*}\right)$ measurements of $\mathrm{LHCb}$ and $\mathrm{B}$-factories with SM theoretical predictions. (c) From [4]. Comparison of the measurements of $R(D)$ and $R\left(D^{*}\right)$ of LHCb, BaBar and Belle with the SM predictions

difference in topology between signal and background is exploited. A cut in the distance between the $B$ vertex and the three pions vertex $(\Delta z)$ is applied at the level of 4 standard deviations, achieving a suppression of the background of three orders of magnitude. After this requirement is applied, the second most important background comes from double charm $B \rightarrow D^{*-} D_{s}^{+}(X)$ decays. In order to reduce this contribution, a requirement has been placed on the output of the multivariate algorithm (Boost Decision Tree, BDT), that has been trained exploiting variables related to the system of the three pions, the $D^{*-} 3 \pi$ system and information from neutral isolation.

In order to obtain the coefficient $\mathcal{K}\left(D^{*-}\right)$, the yields of normalisation and signal channels are extracted. The yield of the normalisation channel is obtained from an unbinned maximum likelihood fit to the $D^{*-} 3 \pi$ invariant mass. The signal yield is obtained from a three-dimensional extended maximum likelihood template fit. In particular, the variables used are the $\tau$ decay time, $t_{\tau}$, the squared transferred momentum, $q^{2}$, and the output of the multivariate algorithm (in high BDT region). The projections from the fit are shown in Fig.2 (a). The result, including the inputs 
from PDG, is $R\left(D^{*-}\right)=0.291 \pm 0.019$ (stat) $\pm 0.026($ syst $) \pm 0.013$ (ext), where the third source of uncertainty comes from the limited knowledge of the branching fractions of the normalisation channel and $\mathcal{B}\left(B^{0} \rightarrow D^{*-} \mu^{+} v_{\mu}\right)$. The main sources of uncertainty are due to the size of simulation sample and the understanding of the spectrum of $D^{*-} 3 \pi$. In particular, there is a considerable uncertainty in establishing the shape of the double charm background and the $D_{s} \rightarrow 3 \pi X$ decay model.

This result of $R\left(D^{*}\right)$ is comparable with other previous measurements, and it is compatible with the SM expectation within $1 \sigma$. The global picture of the previous $R\left(D^{*}\right)$ measurements from $\mathrm{LHCb}$, BaBar and Belle is shown in Fig.2 (b), the world average is in tension with the SM prediction at the level of $2.5 \sigma$. A comparison between the combined measurements of $R(D)$ and $R\left(D^{*}\right)$ from LHCb, BaBar and Belle with the current theoretical predictions is displayed in Fig.2 (c), and leads to a discrepancy of $3.08 \sigma$ between experiment and theory [4].

\section{5. $\mathbf{R}(\mathbf{J} / \psi)$ muonic at $\mathbf{L H C b}$}

The analysis pursued for the $R\left(D^{*}\right)$ can be generalised to the $B_{c}^{+}$sector, yielding the observable $R(J / \psi)[8]$ :

$$
R(J / \psi)=\frac{\mathcal{B}\left(B_{c}^{+} \rightarrow J / \psi \tau^{+} v_{\tau}\right)}{\mathcal{B}\left(B_{c}^{+} \rightarrow J / \psi \mu^{+} v_{\mu}\right)}
$$

The signal is reconstructed using $\tau^{-} \rightarrow \mu^{-} \bar{v}_{\mu} \nu_{\tau}$ and $J / \psi \rightarrow \mu^{+} \mu^{-}$decays. For this decay, the theoretical predictions of the form factors are accompanied by large uncertainties. The yield is obtained by a three-dimensional binned maximum likelihood fit to data. The variables used to fit are the squared missed mass, $m_{m i s s}^{2}, B_{c}^{+}$decay time and $Z$, a variable dependent on the energy of the muon and $q^{2}$. The projections of the fit are shown in Fig.3. The final result is $R(J / \psi)=0.71 \pm 0.17$ (stat) \pm 0.18 (syst), which is in agreement with the SM prediction at the level of $2 \sigma$ [4]. There is room for improvement here, since the Run 2 data will allow finer binning in missing mass, and the systematics uncertainties due to the form factor will be reduced, thanks to new inputs from Lattice QCD.
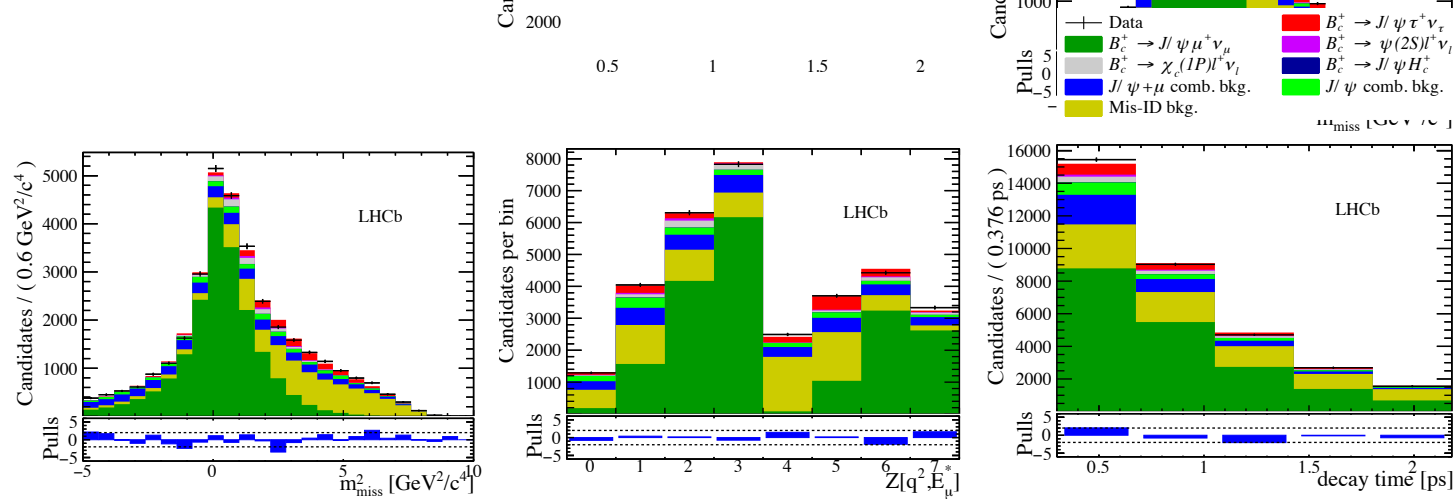

Figure 3: From [8]. Distributions of $m_{m i s s}^{2}$ (left), $\mathrm{Z}$ (middle) and $B_{c}^{+}$decay time (right) of the signal data and the projections of the fit model. The signal distributions are shown in red and the normalisation one in dark green. 


\section{Conclusions}

These proceedings present a review of different LFU tests performed by the LHCb collaboration in semileptonic decays. Tests of LFU have been performed with observables clean from both from the theoretical and experimental point of view. The measurement of these observables are in tension with their SM predictions at the level of 2-3 $\sigma$.

All measurements presented here are performed using LHCb Run 1 data. Therefore, by exploiting Run 2 data, it is expected that both statistical and systematic uncertainties will be reduced. The current status strongly motivates further work both on the theory and on the experimental side to clarify the present observations.

\section{References}

[1] LHCb collaboration, A. A. Alves Jr. et al., The LHCb detector at the LHC, JINST 3 (2008) S08005.

[2] LHCb collaboration, R. Aaij et al., LHCb detector performance, Int. J. Mod. A30 (2015) 1530022, arXiv: 1412.6352 .

[3] The Particle Data Group, C. Patrignani et al., Review of particle physics, Chin. Phys. C40 (2016) 100001, and 2017 update.

[4] HFLAV collaboration, Y. Amhis et al., Averages of b-hadron, c-hadron, and $\tau$-lepton properties as of summer 2018, arXiv: 1919.12524, 2019.

[5] LHCb collaboration, R. Aaij et al., Measurement of the ratio of branching fractions $\mathcal{B}\left(B^{0} \rightarrow\right.$ $\left.D^{*+} \tau^{-} \bar{v}_{\tau}\right) / \mathcal{B}\left(B^{0} \rightarrow D^{*+} \mu^{-} \bar{v}_{\mu}\right)$, Phys. Rev. Lett. 115 (2015) 111803, arXiv: 1506.08614.

[6] LHCb collaboration, R. Aaij et al., Test of lepton flavour universality by the measurement of the $B^{0} \rightarrow D *-\tau^{+} \bar{v}_{\tau}$ branching fraction using three-prong $\tau$ decays, Phys. Rev. Lett. 97 (2018) 072013, arXiv: 1711.02505.

[7] LHCb collaboration, R. Aaij et al., Measurement of the ratio of the $\mathcal{B}\left(B^{0} \rightarrow D^{*-} \bar{v}_{\tau}\right)$ and $\mathcal{B}\left(B^{0} \rightarrow D^{*+} \mu^{-} \bar{v}_{\mu}\right)$ branching fractions using three-prong $\tau$-lepton decays, Phys. Rev. Lett. 120 (2018) 171802, arXiv: 1708.08856.

[8] LHCb collaboration, R. Aaij et al., Measurement of the ratio of branching fractions $B\left(B_{c}^{+} \rightarrow\right.$ $\left.J / \psi \tau^{+} v_{\tau}\right) / B\left(B_{c}^{+} \rightarrow J / \psi \mu^{+} v_{\mu}\right)$, Phys. Rev. Lett. 120 (2018) 121801, arXiv: 1711.05623. 